Check for updates

Cite this: RSC Adv., 2017, 7, 23422

\title{
Sensitive fluorescent detection of fibrin based on the inner filter effect of gold nanoparticles $\uparrow$
}

\author{
Ting Liu, Yuanfu Zhang, (D)* Tingting Hou, Qingwang Xue, Lei Wang \\ and Shuhao Wang $\mathbb{D D}^{*}$
}

A simple, rapid and sensitive fluorescent assay for determination of fibrin has been developed based on the inner filter effect (IFE) of gold nanoparticles (AuNPs). When fibrinogen (Fib), as the precursor of fibrin, was added into the AuNPs solution, the fluorescence of fluorescein was very weak due to the intensive absorption of AuNPs. In the presence of thrombin, Fib was transformed to fibrin which interacted with AuNPs, thereby inducing the aggregation of AuNPs, which induced the recovery of fluorescence. As a result, the present IFE-based approach can detect fibrin ranging from 0.125-2.5 nM with a correlation of 0.9926 . The limit of detection for fibrin was experimentally determined to be $40 \mathrm{pM}$, based on a signal-to-noise ratio $(\mathrm{S} / \mathrm{N})$ of 3 . Notably, the present IFE-based approach had advantages of being simple, time-saving, and economical compared with conventional fluorescent assays. The method is successfully applied to the quantification of fibrin in human plasma samples.

Received 27th February 2017 Accepted 19th April 2017

DOI: $10.1039 / \mathrm{c} 7 \mathrm{ra02422c}$

rsc.li/rsc-advances the method usually needs to covalently link with a fluorophore, which is often time-consuming and expensive, and alters the affinity of a receptor towards the analytes. ${ }^{17-19}$ To overcome these problems, the inner filter effect (IFE)-based fluorescent assays was exploited. ${ }^{20-22}$ The IFE is one of non-irradiation energy conversion models in fluorometry, which refers to the absorption of the excitation and/or emission light of fluorophores by absorbers (quenchers). The IFE is usually considered as an annoying source of error in fluorescent analysis and should be avoided, but it was useful for the design and development of novel probes for highly sensitive fluorescent assay. ${ }^{23-26}$ In contrast to other fluorescence quenching mechanisms, the IFE-based assays have no covalent linking between the absorber and fluorophore, simplifying the synthesis of the fluorescent materials. Meanwhile, it has been reported that IFE-based assays have more sensitivity and selectivity. However, IFE would occur only if the absorption band of the absorber possesses a complementary overlap with the excitation and/or emission bands of the fluorophore. In general, metal ions were not suitable for quenchers in the IFE-based fluorescence assay because of narrow absorption spectrum. Fortunately, gold nanoparticles (AuNPs) have extremely large extinction coefficient and facilely tuned absorption spectrum. AuNPs has been used as quencher in the IFE-based fluorescent assay. ${ }^{27-31}$

In this study, we reported a novel fluorescent assay method to detect the fibrin based on the inner filter effect (IFE) of AuNPs. The principle of this method is illustrated in Fig. 1. Fib is easily adsorbed onto the surface of AuNPs to form Fib-AuNPs. AuNPs was stably dispersed in Fib solution. The fluorescence of fluorescein sodium (FAM) was quenched due to the occurrence
Shandong Provincial Key Laboratory of Chemical Energy Storage and Novel Cell Technology, School of Chemistry and Chemical Engineering, Liaocheng University, Liaocheng 252000, China. E-mail: yuanfu-nice@hotmail.com; shuhaowang@sohu. com; Fax: +86 6358239227

$\dagger$ Electronic supplementary information (ESI) available. See DOI: $10.1039 / \mathrm{c} 7 \mathrm{ra02422 \textrm {c }}$ 


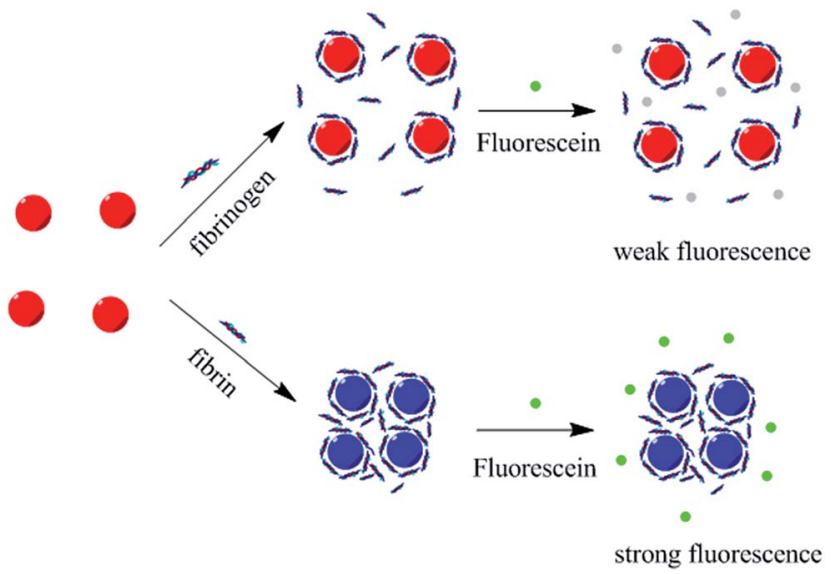

Fig. 1 Schematic representation of the IFE-based fluorescence assay for detection of fibrin.

of IFE, while the absorption band of AuNPs possesses a complementary overlap with the emission bands of FAM. On the other hand, the rigid structure of fibrin induced the aggregation of AuNPs, which then lead to the decreased absorption of AuNPs at $520 \mathrm{~nm}$. As a result, the IFE of AuNPs decreased and the fluorescence increased. Compared to the conventional fluorescence resonance energy transfer (FRET)-based fluorescent assays, AuNPs and fluorophore didn't need to be labeled which offers considerable flexibility and simplicity. It was provided a simple and sensitive approach to detect fibrin.

\section{Experimental}

\section{Chemicals and materials}

Fib was purchased from Sangon. Biotech Co, Ltd. (Shanghai, China, http://www.sangon.com). Chloroauric acid $\left(\mathrm{HAuCl}_{4}\right)$ was purchased from Tianjin Jinbolan Fine Chemicals Co, Ltd. (Tianjin, China, http://www.delanchem.com). Thrombin (1710 U $\mathrm{mg}^{-1}$ ) was obtained from Sigma (St. Louis, MO, USA, http:// www.sigmaaldrich.com/china-mainland/promotions/new.html). Sodium citrate and fluorescein sodium (FAM) were purchased from Aladdin (Shanghai, China, http://www.aladdin-e.com/ zh_cn). Millipore Milli-Q water $\left(18 \mathrm{M} \Omega \mathrm{cm}^{-1}\right)$ was used in all experiments. All other reagents were of analytical grade and used as received, which were purchased from Sinopharm Chemical Reagent Company (Beijing, China, http://www.crc-bj.com).

\section{Apparatus}

All fluorescence measurements were made using Hitachi model F-7000 fluorescence spectrophotometer (Kyoto, Japan, http://www.hitachi.com). Transmission electron microscopy (TEM) measurements were made on a JEM-2100 (Japan Electronics Co., Ltd, http://www.jeol.co.jp/en/products/ detail/JEM-2100F.html). UV-visible adsorption spectra were recorded on a Shimadzu UV-2550 UV/vis spectrophotometer (Kyoto, Japan, http://www.shimadzu.com).

\section{Preparation of AuNPs}

The $13 \mathrm{~nm}$ AuNPs were prepared by citrate-mediated reduction of $\mathrm{HAuCl}_{4}{ }^{32}$ The $50 \mathrm{~mL}$ of $0.04 \% \mathrm{HAuCl}_{4}$ solution was boiled and stirred vigorously. Then $5.6 \mathrm{~mL}$ of $1 \%$ sodium citrate solution was added into the boiling solution. After boiling for $8 \mathrm{~min}$ and followed stirring for $10 \mathrm{~min}$, the solution was cooled to room temperature. Finally it was filtered through a $0.22 \mu \mathrm{m}$ membrane filter and stored in a refrigerator at $4{ }^{\circ} \mathrm{C}$ being used. The size and shape of AuNPs were characterized by a model JEM-2100 transmission electron microscope (TEM, Hitachi). The concentrations of AuNPs were calculated to be $17 \mathrm{nM}$.

\section{Procedure for the detection of fibrin}

The concentration of fibrin was calculated by the enzyme activity of thrombin because pure fibrin hardly existed in the nature. A typical fluorescent analysis was realized by following steps. First, $100 \mu \mathrm{L}$ of $3 \mathrm{nM}$ Fib solution and different concentrations of thrombin ( $5 \mu \mathrm{L}, 50 \mathrm{mM}$ phosphate, $\mathrm{pH} 7.4$ ) were incubated at $37{ }^{\circ} \mathrm{C}$ for 1 hour to obtain fibrin. Second, a $50 \mu \mathrm{L}$ above solution was added into $150 \mu \mathrm{L}$ of $11.3 \mathrm{nM}$ AuNPs solution, the mixture solution was stirred for 7 minutes. Third, a 50 $\mu \mathrm{L}$ above solution was added into $50 \mu \mathrm{L}$ of $1 \mu \mathrm{M}$ fluorescein sodium (FAM) solution, and then the mixed solution was diluted with Tris-HCl buffer (20 mM, pH 7.4) to $200 \mu \mathrm{L}$. The above prepared solution was incubated for $10 \mathrm{~min}$ at room temperature. Finally, the fluorescence intensity of the incubated solution was measured at $520 \mathrm{~nm}$ with an excitation wavelength of $485 \mathrm{~nm}$.

\section{Results and discussion}

\section{Design of the analytical process for fibrin}

The important insight in our design comes from the interaction of AuNPs and fibrin, which are based on the following: (1) Fib can adsorbed onto the surface of AuNPs to enhance the stability of AuNPs. On the other hand, the crosslinking of fibrin induce the aggregation of AuNPs through the polymerization of the unconjugated and conjugated fibrinogen, while Fib was transformed to fibrin in the presence of thrombin; $;^{33-35}$ (2) AuNPs was extraordinarily effective absorber in IFE-based fluorescence assays; (3) the IFE effectively occur while the absorption band of the AuNPs possesses a complementary overlap with the emission bands of the fluorophore (Fig. S1 $\dagger$ ). Fig. 1 depicts the analytical process for fibrin. Fluorescein sodium (FAM) is a manufactured organic dye. It has strong fluorescence emission maximum of $520 \mathrm{~nm}$. It is widely used as a fluorescent tracer for many applications. While Fib easily adsorbed onto the surface of AuNPs to form Fib-AuNPs. The fluorescence of fluorescein is very weak due to the intensive absorption of AuNPs (Fig. 1). In the presence of fibrin, the fibrin made AuNPs crosslinked and rapidly induced the aggregation of AuNPs, which thereby leads to the decreased absorption of AuNPs. The IFE-decreased emission of fluorescein is recovered (Fig. 2). The concentration of fibrin could be measured through the recovery of fluorescence at $520 \mathrm{~nm}$. 


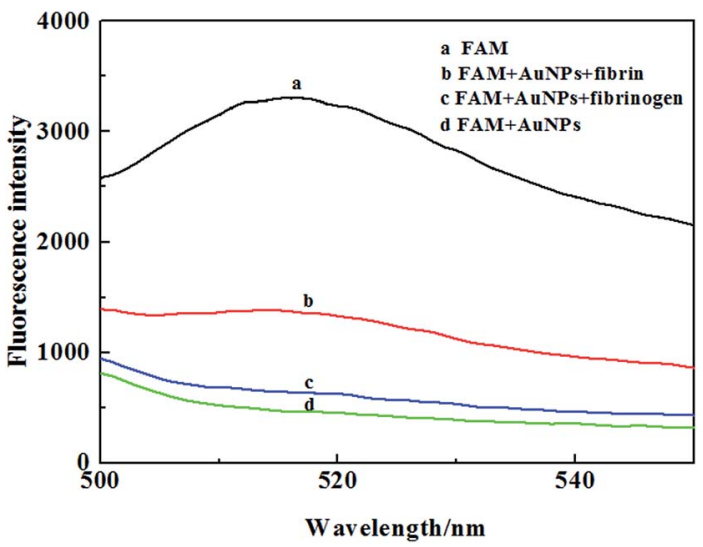

Fig. 2 Fluorescence emission spectra of FAM in the different system. Experimental conditions: AuNPs $11.3 \mathrm{nM}$, FAM $1 \mu \mathrm{M}$, Fib $3 \mathrm{nM}$, fibrin $3 \mathrm{nM}$.

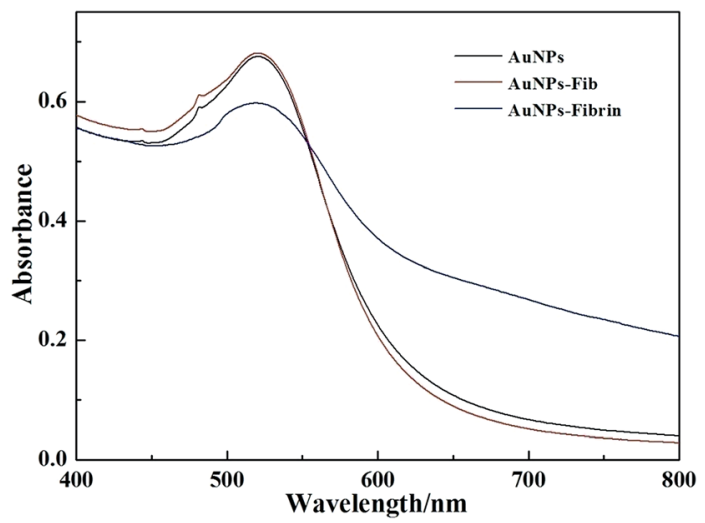

Fig. 3 UV/vis absorption spectra of AuNPs stabilized by Fib and fibrin.

UV/vis spectroscopy (Fig. 3) was used to explore the change of AuNPs state induced by Fib/fibrin in this system. The AuNPs display a maximal absorption band at $520 \mathrm{~nm}$. In the presence of Fib, there still was an absorption band at $520 \mathrm{~nm}$, and the absorption band of the AuNPs overlapped the emission bands of the fluorophore. The IFE effectively occurred and the fluorescence of fluorescein decreased. On the other hand, the UVvisible spectrum displays the characteristic red shift and broadening of the surface plasmon band in the presence of fibrin, indicating that the AuNPs are crosslinked and aggregated. Thereby, the IFE-decreased emission of fluorescein is recovered. In order to know the microstructure of the AuNPs with fibrin, the TEM images were obtained. As shown in Fig. S2, $\dagger$ AuNPs reveal uniform particles with an average size of $\sim 13 \mathrm{~nm}$ in diameter. In the presence of Fib, the AuNPs are mono-dispersed, whereas the AuNPs aggregate together in the presence of fibrin. The results were consistent with the change of the UV-visible absorption spectra.

\section{Optimization of the variables of the measuring system}

In order to study the effect of AuNPs particle size on analytical performance of fibrin, four different nanometer-sized AuNPs
(2.6 nm, $13 \mathrm{~nm}, 20 \mathrm{~nm}$ and $40 \mathrm{~nm}$ ) were prepared. The experimental results showed that $13 \mathrm{~nm}$ AuNPs were more sensitive to the fibrin (Fig. S3†) than the other three AuNPs. The reason is possibly that the size of AuNPs didn't only influence the fluorescence quenching ability of AuNPs, but also affected the FibAuNPs binding. In this paper, because the absorption band of AuNPs need to overlap with the emission bands of the fluorescein. From Fig. S4, $\dagger$ it was known that the AuNPs (13-20 nm) displayed maximal absorption band at $520 \mathrm{~nm}$ which was according with the design of sensing well. In addition, the stoichiometry between fibrinogen and AuNPs was dependent on particle size. ${ }^{36}$ While the size of AuNPs was larger than $13 \mathrm{~nm}$, AuNPs bound multiple fibrinogen molecules, which can reduce the sensitivity of this method. While the size of AuNPs was smaller than $13 \mathrm{~nm}$, maximum binding of Fib-AuNPs occurred at approximately 1 fibrinogen per 2 AuNPs, which easily induced the aggregation of AuNPs. Only for the $13 \mathrm{~nm}$ AuNPs, maximum binding occurred at $1: 1$ stoichiometry, which better accorded with the design of sensing. ${ }^{36,37}$ We also investigated the effect of AuNPs concentration on the fluorescence response (Fig. S5 $\dagger$ ). The experimental results showed that low concentration of AuNPs could enhance the response for fibrin, but the background fluorescence was high and the fluorescent restoration was not obvious. Otherwise high concentration of AuNPs could decrease the background fluorescence, but AuNPs concentration was so high that fluorescent restoration was quenched. While the concentration of AuNPs was $11.3 \mathrm{nM}$, fluorescent restoration $(\Delta F)$ reached biggest. So we used $11.3 \mathrm{nM}$ of AuNPs (13 nm) for all experiments.

In this work, reaction time is a key factor for reaction, which is characterized with quenching and aggregation kinetic. There were two action times including the incubation time of AuNPsfibrin (Fig. S6 $\dagger$ ) and AuNPs-FAM (Fig. S7†). With the increasing of the incubation time of AuNPs-fibrin, the $\Delta F$ rose quickly and reached a maximum in the period of 7 minutes. After in addition of FAM, the AuNPs-FAM mixture was incubated for $10 \mathrm{~min}$, and the $\Delta F$ reached a maximum. So, we chose 7 minutes and $10 \mathrm{~min}$ as the incubation time of AuNPs-fibrin and AuNPs-FAM.

\section{Sensitivity and selectivity}

Under the optimized conditions, the quantitative behavior of the fluorescent assay was assessed with different concentrations of fibrin. A good linear relationship between the increased fluorescence intensity $(\Delta F), \Delta F=F-F_{0}$, where $F$ and $F_{0}$ are the fluorescence intensity of the system at $520 \mathrm{~nm}$ in the presence of fibrin or Fib, respectively. As shown in Fig. 4, the fluorescence intensity increased with the increasing fibrin concentration, to reveal a linear relationship in the fibrin concentration range $0.125-2.5 \mathrm{nM}$. The regress equation is $\Delta F=360.7+92.7 c(c$ is the concentration of fibrin, $\mathrm{nM}$ ) with a correlation coefficient of 0.9989. The relative standard deviation (R.S.D.) for $2 \mathrm{nM}$ fibrin measurement is $2.7 \%(n=11)$. The detection limit is taken to be three times of the standard derivation in the blank solution was found to be $40 \mathrm{pM}$.

In our work, bovine serum albumin (BSA), thrombin, IgG, glucose oxidase (GOD) and lysozyme were selected to study the 


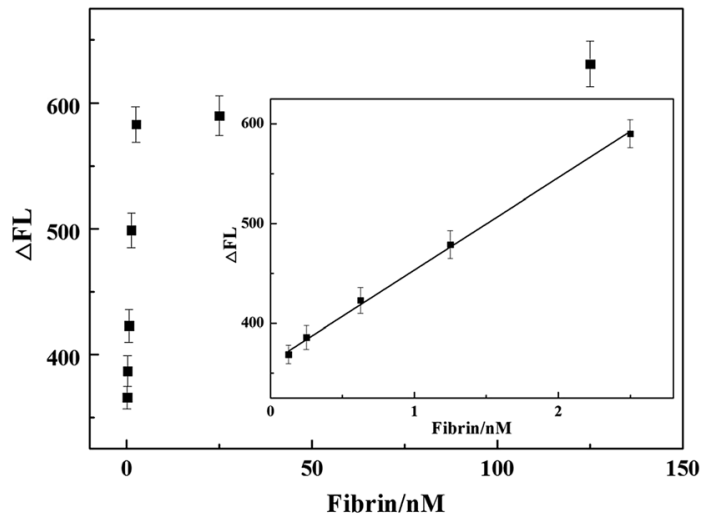

Fig. 4 The difference value of fluorescence intensity $(\Delta F)$ profiles of the assay for different concentration of fibrin. Inset shows a linear relationship between $\Delta F$ and the fibrin concentration $(C)$ from $0.125 \mathrm{nM}$ to $2.5 \mathrm{nM}$. Experimental conditions: AuNPs $11.3 \mathrm{nM}, \mathrm{FAM} 1$ $\mu \mathrm{M}$.

specificity of the method. As shown in Fig. 5, while the concentration of interfering proteins was 400-fold higher than that of fibrin, the experimental results showed that a significant fluorescent increase was observed for fibrin and the fluorescent restoration $(\Delta F)$ is biggest. However, other proteins had little influence on the $\Delta F$. The results revealed this facile fluorescence method for fibrin detection was sensitive and effective.

\section{Detection of fibrin in human plasma with the proposed method}

The proposed method was used to detect fibrin in human plasma. The samples from some volunteers were collected from Liaocheng People's Hospital. Previously the fresh serum samples were first analyzed by the ELISA method (UnicelDXC800, Beckman Coulter auto-analyzer) in Liaocheng People's Hospital. And then, the samples were re-assayed with the proposed method. Fresh serum samples were centrifugated at $5400 \mathrm{rcf}$ for $10 \mathrm{~min}$. The supernatant was saved. All the

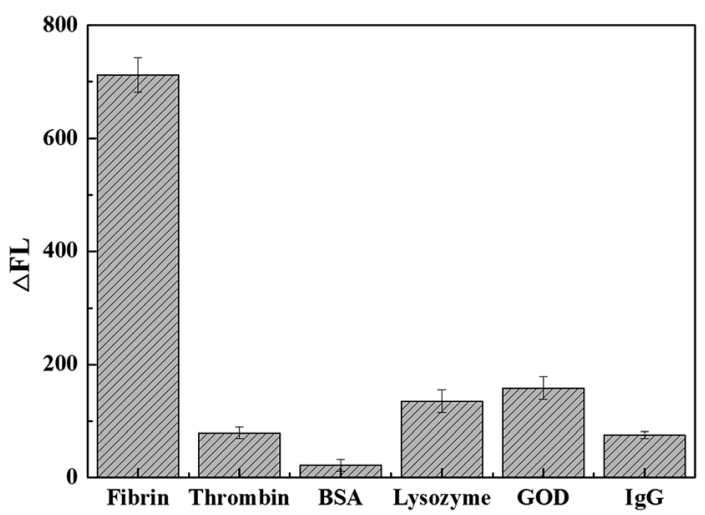

Fig. 5 Selectivity of the IFE-based fibrin fluorescence assay over several molecules: fibrin $(2.5 \mathrm{nM})$, thrombin $(1 \mu \mathrm{M})$, BSA $(1 \mu \mathrm{M})$, lysozyme $(1 \mu \mathrm{M}), \mathrm{GOD}(1 \mu \mathrm{M})$ and $\lg G(1 \mu \mathrm{M})$. Experimental conditions: AuNPs 11.3 nM, FAM $1 \mu \mathrm{M}$.
Table 1 Determination of fibrin in human plasma

\begin{tabular}{|c|c|c|c|c|}
\hline Sample & $\begin{array}{l}\text { Conventional } \\
\text { method (nM) }\end{array}$ & $\begin{array}{l}\text { Proposed method } \\
(\mathrm{nM})\end{array}$ & $\begin{array}{l}\text { Added } \\
(\mathrm{nM})\end{array}$ & $\begin{array}{l}\text { Recovery } \\
(\%)\end{array}$ \\
\hline \multirow[t]{2}{*}{1} & \multirow[t]{2}{*}{0.671} & \multirow[t]{2}{*}{0.646} & 0.300 & 97 \\
\hline & & & 0.500 & 95 \\
\hline \multirow[t]{2}{*}{2} & \multirow[t]{2}{*}{0.954} & \multirow[t]{2}{*}{0.983} & 0.300 & 98 \\
\hline & & & 0.500 & 103 \\
\hline \multirow[t]{2}{*}{3} & \multirow[t]{2}{*}{1.523} & \multirow[t]{2}{*}{1.500} & 0.300 & 104 \\
\hline & & & 0.500 & 101 \\
\hline \multirow[t]{2}{*}{4} & \multirow[t]{2}{*}{1.022} & \multirow[t]{2}{*}{1.041} & 0.300 & 96 \\
\hline & & & 0.500 & 97 \\
\hline \multirow[t]{2}{*}{5} & \multirow[t]{2}{*}{0.814} & \multirow[t]{2}{*}{0.792} & 0.300 & 96 \\
\hline & & & 0.500 & 105 \\
\hline \multirow[t]{2}{*}{6} & \multirow[t]{2}{*}{0.763} & \multirow[t]{2}{*}{0.754} & 0.300 & 103 \\
\hline & & & 0.500 & 98 \\
\hline
\end{tabular}

samples were previously diluted in an appropriate extension (1:50 000) to fit the concentration of the analyte within the linear range of the calibration curve. All experiments were performed in compliance with the guideline "Biomedical research ethics review method involving people" (China), and approved by the medical ethics committee at Liaocheng People's Hospital. Informed consents were obtained from human participants of this study. The results (Table 1 in the ESI $\dagger$ ) showed good agreement with the expected and observed values (Table 1).

\section{Conclusion}

In conclusion, we have demonstrated the design of a novel, simple, and sensitive fluorescent method for detecting fibrin based on IFE of AuNPs. Because the absorption band of AuNPs possesses a complementary overlap with the emission bands of FAM, the fluorescence of FAM was quenched due to the occurrence of IFE. In the presence of fibrin, fibrin interacted with the AuNPs, which induced the aggregation of AuNPs and decreased the absorption of AuNPs at $520 \mathrm{~nm}$. Thereby, the IFE of AuNPs decreased and the fluorescence emission of FAM was restored significantly. Under optimized conditions, a high sensitivity towards fibrin was achieved with a detection limit as low as 40 pM. Compared with conventional fluorescent assays, the present IFE-based strategy has several advantages including simple, time-saving, and economical. Therefore, the proposed fluorescent assay has great potential for protein assay in the fields of molecular biology and clinical diagnostics.

\section{Acknowledgements}

This work was funded by the Natural Science Foundation of China (No. 21675072), the Natural Science Foundation of Shandong Province (No. ZR2013BQ016).

\section{References}

1 M. W. Mosesson, K. R. Siebenlist and D. A. Meh, Ann. N. Y. Acad. Sci., 2001, 936, 11-30. 
2 M. W. Mosesson, J. Thromb. Haemostasis, 2005, 3, 1894-1904. 3 J. Calcaterra, K. E. Van Cott, S. P. Butler, G. C. Gil, M. Germano, H. A. van Veen, K. Nelson, E. J. Forsberg, M. A. Carlson and W. H. Velander, Biomacromolecules, 2013, 14, 169-178.

4 T. Lei, X. Zhao, S. Jin, Q. Meng, H. Zhou and M. Zhang, Clin. Genitourin. Cancer, 2013, 11, 56-62.

5 P. Gromov, J. M. A. Moreira, I. Gromova and J. E. Celis, Clin. Proteomics, 2006, 2, 45-65.

6 S. Yakovlev, S. Gorlatov, K. Ingham and L. Medved, Biochemistry, 2003, 42, 7709-7716.

7 M. E. Martinuzzo, C. Ujhelly, L. H. Barrera, M. A. D'Adamo, M. S. López, J. C. Otaso and J. Oyhamburu, Clin. Lab., 2016, 62, 2085-2089.

8 H. Aizawa, S. Kurosawa, M. Tozuka, J.-W. Park, K. Kobayashi and H. Tanaka, Biosens. Bioelectron., 2003, 18, 765-771.

9 A. Hamano, S. Tanaka, Y. Takeda, M. Umeda and Y. Sakata, Clin. Chim. Acta, 2002, 318, 25-32.

10 A. Hamano, M. Umeda, Y. Ueno, S. Tanaka, J. Mimuro and Y. Sakata, Clin. Chem., 2005, 51, 183-188.

11 K. Okajima, M. Uchiba, K. Murakami, H. Okabe and K. Takatsuki, Am. J. Hematol., 1996, 51, 186-191.

12 J. Koopman, F. Haverkate, P. Koppert, W. Nieuwenhuizen, E. J. Brommer and W. G. Van der Werf, J. Lab. Clin. Med., 1987, 109, 75-84.

13 H. Aizawa, S. Kurosawa, M. Tozuka, J. W. Park and K. Kobayashi, Sens. Actuators, B, 2004, 101, 150-154.

14 Y. Zhou, J. F. Zhang and J. Yoon, Chem. Rev., 2014, 114, 55115571.

15 L. Yuan, W. Lin, K. Zheng, L. He and W. Huang, Chem. Soc. Rev., 2013, 42, 622-661.

16 K. Li and B. Liu, Chem. Soc. Rev., 2014, 43, 6570-6597.

17 J. Zhou, X. Xu, W. Liu, X. Liu, Z. Nie, M. Qing, L. Nie and S. Yao, Anal. Chem., 2013, 85, 5746-5754.

18 C. Ma, S. Liu and C. Shi, Biosens. Bioelectron., 2014, 58, 5760.
19 B.-C. Yin, Y.-Q. Liu and B.-C. Ye, One-step, J. Am. Chem. Soc., 2012, 134, 5064-5067.

20 X. Cui, M. Liu and B. Li, Analyst, 2012, 137, 3293-3299.

21 J. Li, X. Li, X. Shi, X. He, W. Wei, N. Ma and H. Chen, ACS Appl. Mater. Interfaces, 2013, 5, 9798-9802.

22 L. Xu, B. Li and Y. Jin, Talanta, 2011, 84, 558-564.

23 G. Li, H. Fu, X. Chen, P. Gong, G. Chen, L. Xia, H. Wang, J. You and Y. Wu, Anal. Chem., 2016, 88, 2720-2726.

24 M. Zheng, Z. Xie, D. Qu, D. Li, P. Du, X. Jing and Z. Sun, ACS Appl. Mater. Interfaces, 2013, 5, 13242-13247.

25 N. Shao, Y. Zhang, S. Cheung, R. Yang, W. Chan, T. Mo, K. Li and F. Liu, Anal. Chem., 2005, 77, 7294-7303.

26 H. Kim, B.-I. Lee and S.-H. Byeon, Chem. Commun., 2015, 51, 725-728.

27 J. Li, X. Li, X. Shi, X. He, W. Wei, N. Ma and H. Chen, ACS Appl. Mater. Interfaces, 2013, 5, 9798-9802.

28 X. Yan, H. Li, X. Han and X. Su, Biosens. Bioelectron., 2015, 74, 277-283.

29 L. Shang and S. Dong, Anal. Chem., 2009, 81, 1465-1470.

30 X. Yan, H. Li, Y. Li and X. Su, Anal. Chim. Acta, 2014, 852, 189-195.

31 H.-C. Chang and J.-A. Ho, Anal. Chem., 2015, 87, 1036210367.

32 Y. Zhang, J. Liu, T. Liu, H. Li, Q. Xue, R. Li, L. Wang, Q. Yue and S. Wang, Biosens. Bioelectron., 2016, 77, 111-115.

33 C.-K. Chen, C.-C. Huang and H.-T. Chang, Biosens. Bioelectron., 2010, 25, 1922-1927.

34 A. A. Shemetov, I. Nabiev and A. Sukhanova, ACS Nano, 2012, 6, 4585-4602.

35 J.-W. Jian and C.-C. Huang, Chem.-Eur. J., 2011, 17, 23742380.

36 Z. J. Deng, M. Liang, I. Toth, M. J. Monteiro and R. F. Minchin, ACS Nano, 2012, 6, 8962-8969.

37 Y. Niu, P. Wang, Y. Zhao and A. Fan, Analyst, 2013, 138, 1475-1482. 